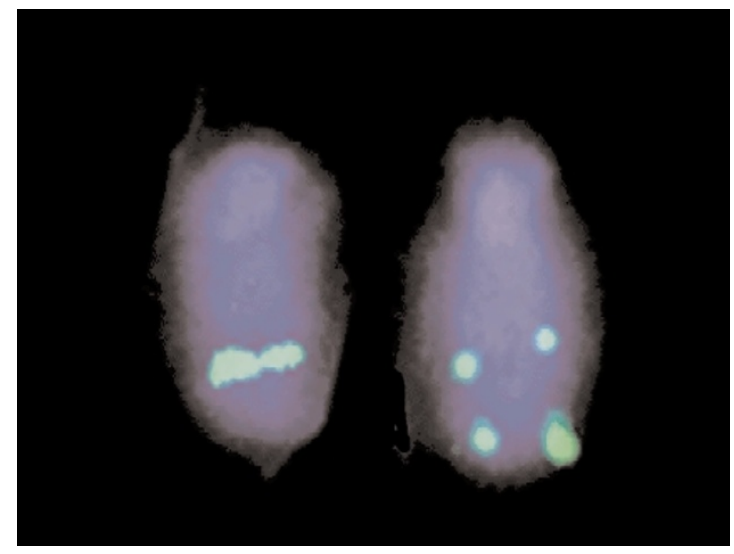

\title{
Does duplicity make you anxious?
}

Anxiety disorders such as panic disorder and agoraphobia - which might afflict up to $20 \%$ of people during their lifetime - are complex diseases that result from the combined effects of many loci and their interaction with the environment. Although genetic susceptibility determinants have been isolated for some complex disorders, such as Crohn disease (see July's Highlights), those for psychiatric illnesses have been harder to pin down. Gratacòs and colleagues now report the first instance of a genomic variant associated with susceptibility to panic and phobic disorders: a duplication on human chromosome 15 that arises by a new mutational mechanism.

Based on the observation - made ten years ago by Bulbena et al. - that patients with joint laxity, or hypermobility, were four times more likely to suffer from anxiety disorders than the general population, the authors decided to investigate whether the psychiatric and the joint phenotypes had a common genetic basis. Indeed, of the seven extended families with anxiety disorders they studied, $\sim 64 \%$ of individuals also had joint laxity.
A preliminary cytogenetic study revealed a putative alteration on chromosome 15 in all samples, which was identified as an interstitial duplication of $\sim 17 \mathrm{Mb}$ at 15q24-26 (DUP25). In a subsequent scaled-up study, the duplication was seen in $90 \%$ of patients with one or more anxiety disorders, and in $80 \%$ of subjects with joint laxity, but only in $7 \%$ of controls. The association of DUP25 with anxiety was found in sporadic, as well as in familial, cases. Because the orientation and position of the duplication varies between individuals from the same family - and even between cells from the same individual — the authors believe the duplication is transmitted in a non-Mendelian fashion. Instead, they propose that illegitimate mitotic recombination events might occur that are mediated by low-copy repeats that flank the DUP25. Changes in dosage of one or more of the 59+ genes in DUP25 might contribute to the clinical phenotype.

This study provides not only an explanation for the association between anxiety disorders and joint laxity, but also the first evidence of genetic factors that influence suceptibility to a
A normal (right) and a DUP25 chromosome 15 (left) from an with an inverted telomeric duplication. Courtesy of Marga Nadal and Xavier Estivill, MMGC-IRO, Barcelona, Spain.

major psychiatric illness. Getting to the crucial gene or genes, the dosage of which is disrupted in DUP25, will not be easy, as the nonMendelian transmission of DUP25 precludes a straightforward linkage analysis. The authors are optimistic that the improved diagnostic tools using DUP25 as a marker and the association with joint laxity — will help in this effort. Mouse models of DUP25 will also be essential for finding out how these disorders develop and for discovering possible future treatments.

Tanita Casci

\section{(2) References and links}

ORIGINAL RESEARCH PAPER Gratacòs, M. et al

A polymorphic genomic duplication on human chromosome 15 is a susceptibility factor for panic and phobic disorders. Cell 106, 367-379 (2001)

FURTHER READING Emanuel, B. S. \& Shaikh, T. H. Segmental duplications: an 'expanding' role in genomic instablility and disease. Nature Rev. Genet. 2, 791-800 (2001) | Bulbena, A. et al. Joint hypermobility syndrome and anxiety disorders. Lancet $\mathbf{2}$. 694 (1988)

\section{EVOLUTION}

\section{Separating the old from the new}

The conserved family of homeobox (Hox) genes is well known for specifying patterning along the metazoan anterior-posterior axis. Equally ancient is the peculiar correspondence between the order of the Hox genes on the chromosome and their expression domains along the body axis, known as collinearity. With the emergence of novel morphological structures, however, some Hox genes have been recruited for functions other than axial specification - limb development in vertebrates, for example. For these genes, the collinearity rule is not always respected, with the genes being controlled instead by shared enhancers. By molecularly dissecting the mouse and human Hoxd clusters, François Spitz and colleagues have shown that collinear expression is dictated by control elements within or close to the Hoxd cluster, whereas non-collinear expression is under the control of more remote elements.

The proteins encoded by the nine genes in the mouse and human Hoxd cluster are highly conserved. In the first of two approaches to map the regulatory potential of this cluster, the authors monitored the expression and functional properties of the human $H O X D$ cluster when introduced into mice on a $120-\mathrm{kb}$ PAC. The human HOXD transcripts recapitulated the expression of their mouse counterparts, and functionally rescued the trunk phenotypes of inactive mouse Hoxd11-13 genes. Significantly, however, no phenotypic rescue of the developing limbs occurred, nor was human HOXD expression present in the limb domain, indicating that the elements required for limb expression (and in some other organs) lie outside the $120-\mathrm{kb}$ genomic fragment. A similar effect was observed when a larger $215-\mathrm{kb}$ construct containing the mouse Hoxd cluster was used. The remote location of the enhancers required for Hox gene expression in the limb was confirmed by generating nested deletions of the mouse Hoxd cluster and following the expression of a Hoxd11-lacZ reporter gene that replaced the deleted sequences. Although the progressive removal of the cluster led to the loss of lacZ expression in the trunk as expected, its expression in the limb was unaffected, even when the entire Hoxd cluster was deleted.

This study shows that Hox expression domains can be divided into those that reflect ancient functions, which are controlled by sequences within the cluster itself, and others that have been recruited for new functions, which depend on enhancers outside the cluster (at least $100 \mathrm{~kb}$ upstream from Hoxd, the authors propose). The recruitment of Hox genes to new capacities required the capture or formation of new control elements; their remote location can probably be explained by the difficulty of incorporating new regulatory sequences into the tightly organized Hox cluster without disturbing the finely honed assembly of this ancient module.

Tanita Casci

(9) References and links ORIGINAL RESEARCH PAPER Spitz, F. et al. Large scale transgenic and cluster deletion analysis of the HoxD complex separate an ancestral regulatory module from evolutionary innovations. Genes Dev. 15, 2209-2214 (2001) WEB SITE

Denis Duboule's lab:

http://www.unige.ch/sciences/biologie/biani/duboule/ index_st.htm 